\title{
Adaptive load-based and channel-aware power saving for non-real-time traffic in LTE

\author{
Chun-Chuan Yang ${ }^{1}$, Jeng-Yueng Chen ${ }^{2^{*}}$, Yi-Ting Mai ${ }^{3}$ and Ching-Hsiang Liang
}

\begin{abstract}
The popularity of handheld devices, which are usually powered by batteries, has made power saving an important and practical issue in recent years. Techniques of power saving for user devices using mobile communication systems such as WiMAX and LTE (Long-Term Evolution) are parts of the major focuses in the literature. In this paper, two revised schemes of the authors' previously proposed power saving schemes for IEEE 802.16 are proposed to be applied in LTE. The proposed schemes, namely LTE-LBPS-Aggr and LTE-LBPS-Merge, estimate the input load by traffic measurement and the channel capacity by channel quality indicator (CQI) reports, calculate the length of the sleep cycle, and notify related user equipments (UEs) of the next radio-on time for receiving data. The difference between LTE-LBPS-Aggr and LTE-LBPS-Merge lies in the grouping of UEs for sleep scheduling. LTE-LBPS-Aggr treats all UEs in a group, while LTE-LBPS-Merge allows multiple groups of UEs in sleep scheduling. The simulation study shows that in comparison with standard-based mechanisms, the proposed schemes can achieve better power saving efficiency at the cost of moderate increase on delays and the signaling overhead.
\end{abstract}

\section{Introduction}

With the increasing popularity of all sorts of mobile devices and cloud computing applications [1], modern life is being brought into a new era of mobile communications in recent years. To address the intense demand, the wireless technology for the fourth generation (4G) [2] of mobile broadband communications is standardized. 4G candidate systems including Mobile WiMAX [3] and LTE (Long-Term Evolution) [4, 5] are commercially deployed. The LTE standard is defined and supported by all major players in the telecommunication industry and is backward compatible with GSM/UMTS cellular systems, which makes LTE deployment easier than Mobile WiMAX, giving LTE benefit over its competitors in the $4 \mathrm{G}$ market.

However, based on GSM/EDGE and UMTS/HSPA network technologies, both the core network (Evolved Packet Core, EPC) and the radio access (Evolved Universal Terrestrial Radio Access Network, E-UTRAN) in LTE are fully packet-switched, rather than following the circuit-switched model of earlier systems. LTE is designed to work with a variety of different bandwidths

\footnotetext{
* Correspondence: jychen@ieee.org

${ }^{2}$ Department of Information Networking Technology, Hsiuping University of Science and Technology, Taichung City, Taiwan

Full list of author information is available at the end of the article
}

and to deliver a peak data rate of $100 \mathrm{Mbps}$ in the downlink and $50 \mathrm{Mbps}$ in the uplink. The enhanced version of LTE, namely LTE-Advanced (LTE-A), is designed with advanced features to deliver a peak data rate of $1000 \mathrm{Mbps}$ in the downlink and $500 \mathrm{Mbps}$ in the uplink. The specifications for LTE produced by the Third Generation Partnership Project (3GPP) are organized into releases, each of which contains a stable and clearly defined set of features. LTE was first introduced in Release 8 [4], and initial enhancements were included in Release 9. The extra capabilities required for LTE-A were specified in Release 10 [5]. As the specification of Release 11 of the LTE standards is approaching its completion, 3GPP is gradually moving its focus toward the next major step in the evolution of LTE (Release 12).

The progress of modern radio communication techniques has been driven by the academic research in a variety of areas, such as wireless mesh networks [6], vehicular networks [7], cognitive radio [8,9], efficient use of radio resource $[10,11]$, and heterogeneous/composite radio networks [12-14]. One of the research issues in mobile communications is power saving/management at the user side as well as at the network side. Power saving at the network side creates the benefit of energy cost reduction, but the user side is more critical since the user device is usually battery-powered and the length of the operational

\section{Springer}

(c) 2015 Yang et al. Open Access This article is distributed under the terms of the Creative Commons Attribution 4.0 International License (http://creativecommons.org/licenses/by/4.0/), which permits unrestricted use, distribution, and reproduction in any medium, provided you give appropriate credit to the original author(s) and the source, provide a link to the Creative Commons license, and indicate if changes were made. 
time in communications is always a main focus. Discontinuous Reception mode (DRX) $[15,16]$ is supported to conserve the power of the mobile terminal, namely the user equipment (UE) in LTE. The UE powers down most of its circuitry in DRX when there are no packets to be transmitted or received. During this time, the UE listens to the downlink (DL) occasionally and may not keep in sync with uplink (UL) transmission depending on its RRC (Radio Resource Control) state. There are additional advantages in using DRX, such as radio link resource saving on both UL and DL to increase system capacity.

The authors have been working on the issue of power saving in IEEE 802.16 for some years. The idea of LoadBased Power Saving (LBPS) and associated schemes were proposed to adaptively schedule the sleep (radio-off) time of the user device for the current network load [17]. Extension of LBPS to integrate the user side and the network side in sleep scheduling was also proposed [18].

In order to make LBPS work for LTE, the mechanism of channel capacity estimation by channel quality indicator (CQI) reporting as well as two revised LBPS schemes, namely LTE-LBPS-Aggr and LTE-LBPS-Merge, are proposed in this paper. A simulation study shows that the two proposed schemes can adapt to the fluctuated channel capacity as well as the input load and achieve much higher power saving efficiency in comparison with standard-based mechanisms. The remainder of the paper is organized as follows: In Section 2, a survey of the standard DRX in LTE, DRX related work, and our previous work of LBPS in IEEE 802.16 is presented. Two revised LBPS schemes for LTE are presented in Section 3. Results of performance evaluation are presented in Section 4. Finally, Section 5 concludes this paper.

\section{Related work}

In this section, the standardized operation of LTE DRX is briefly introduced, and a survey of related research results for the issue of LTE DRX and power saving is presented. Lastly, the authors' previous work of LBPS is presented.

\subsection{LTE DRX}

In LTE, the DRX mode can be enabled in both of the following states of the radio link between the UE and the base station (called eNodeB or eNB): RRC_idle and RRC_connected. As illustrated in Fig. 1, a DRX cycle consists of an "Opportunity for DRX period" (radio off) during which the UE can skip reception of DL channels and an "ON duration" (radio on) during which the UE should monitor the physical downlink control channel $(\mathrm{PDCCH})$ to identify DL data. The inactivity timer is used to trigger the start of a DRX cycle in the RRC_connected state. The parameterization of the DRX cycle involves a trade-off between battery saving and the access latency. A long Opportunity for DRX period is beneficial for lengthening the UE's battery life. On the other hand, a shorter DRX period is better for faster response. Therefore, two DRX cycles, namely Short DRX Cycle and Long DRX Cycle, can be configured for each UE. The transition between the Short DRX Cycle and the Long DRX Cycle can be controlled by the DRX Short Cycle Timer or by explicit commands from eNB.

\subsection{Related research}

LTE DRX research in the literature can be classified into the following categories: modeling for performance analysis, optimized selection of DRX parameters, traffic characteristic-based heuristic mechanisms, and miscellaneous schemes. In [19-21], the DRX operation with

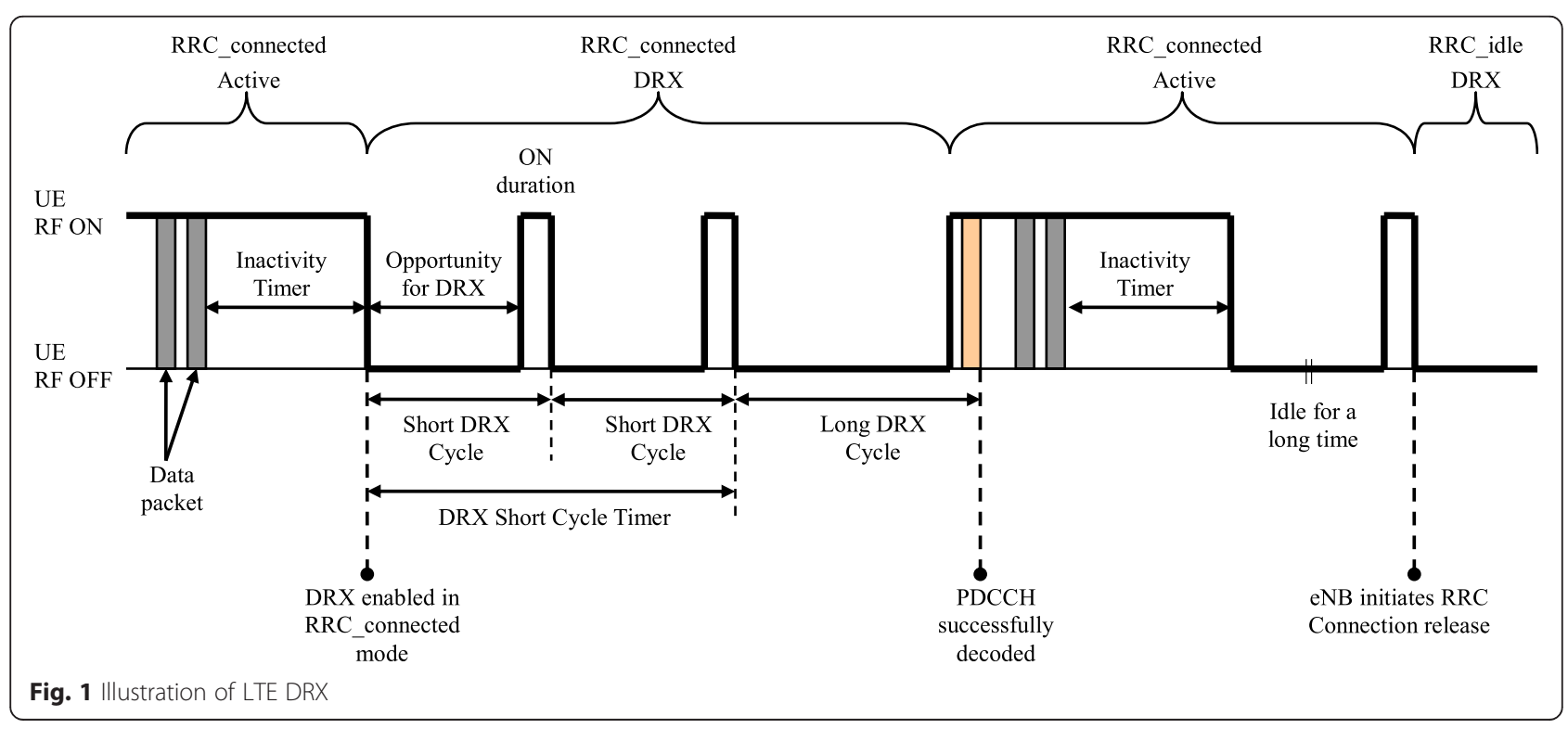


bursty traffic was modeled by a semi-Markov process to derive the equations of power saving and delay performance. Authors in [22] adopted an approach of dividing the DRX operation into several independent parts, which was claimed to be easier for theoretical analysis of accurate power saving factor and packet transmission delay.

Optimized selection of DRX configuration is concerned about the trade-off between power saving efficiency and delay performance. Authors of [23] employed a partially observable Markov decision process to determine the optimal selection of DRX parameters by maximizing power saving efficiency under the delay constraint. A series of research dealing with the trade-off of the two conflicting performance metrics was proposed in [24-26]. In [27], the bustiness of the traffic was considered and combinations of DRX parameters were evaluated in terms of user throughput, power consumption, and network performance to select the best DRX configuration. The impact of DRX operation on the QoS performance of VoIP traffic under dynamic and semi-persistent packet scheduling strategies was studied in [28]. Traffic characteristics of mobile Internet applications were explored, and the impact of the characteristics on LTE device power and air interface signaling was investigated in [29].

Heuristic mechanisms for adjusting the inactivity timer based on CQI reports from the users were proposed in [30], in which a multi-threshold adaptive configuration DRX mechanism, namely M-ADRX, was designed. In [31], the effects of Short DRX Cycle and Long DRX Cycle on power consumption for Voice and Web traffic were investigated, and the influences of the length of TTI (time transmission interval) on power saving efficiency and access delay were evaluated. An analysis of processing in LTE L2 and L3 protocols for frequent signaling procedure was presented in [32], in which four signaling procedures, namely Attach, Idle-Connected, Handover, and Tracking Area Update, were analyzed.

Lastly, not too many research papers in the literature targeted on power saving at the side of eNB in LTE. Authors in [33] showed that energy consumption can be significantly reduced by introducing discontinuous transmission (DTX) at eNB, and up to $61 \%$ of the energy can be saved in a realistic traffic scenario. A distributed wake-up scheduling algorithm for the base transceiver stations in a cellular network was proposed in [34], in which a base transceiver station dynamically decides on its operation mode (off, sleep, or active) according to the measured traffic load of itself and its neighbors in a distributed manner.

\subsection{Previous work of LBPS}

The objective of LBPS is to adaptively adjust the sleep window size of each MSS (mobile subscriber station) in IEEE 802.16 to better fit in current traffic load. The base station (BS) in LBPS estimates the current load for each
MSS (denoted by bits per time frame) by collecting and exponentially averaging the samples of load measure. Although uplink traffic can also be integrated into LBPS schemes, only downlink traffic is considered in the following for presentation purpose. LBPS sets a target threshold of data accumulation in the buffer for an MSS and dynamically calculates its next sleep window size. In this way, LBPS can adapt to different traffic loads and still achieves a proper level of power saving. The basic version of LBPS is called LBPS-Aggr, in which all the traffic in the network is treated as an aggregate flow in calculating the size of the sleep window. In LBPS-Aggr, the traffic arrival process is assumed to be Poisson, and data accumulation under load $\lambda$ in a time frame is calculated by the following equation:

$$
\begin{aligned}
& \operatorname{Prob}[i \text { bits arrived in a time frame }]=\frac{e^{-\lambda T}(\lambda T)^{i}}{i !}, \\
& \text { where } T \text { is the length of a time frame }
\end{aligned}
$$

The threshold of data accumulation is denoted by Data_TH (bits), which is practically set as the capacity of a time frame. The probability of data accumulation exceeding Data_TH packets over $K$ time frames in a row, denoted by $P_{\text {Acc }}\left(K, D a t a_{-} T H\right) \equiv$ Prob [\# of bits arrived in $K$ time frames $>$ Data_TH], can be calculated as follows:

$$
\begin{aligned}
P_{\text {Acc }}(K, \text { Data_TH }) & =\sum_{\substack{i=\text { Data_TH+1 } \\
\text { Data_TH }}}^{\infty} \frac{e^{-\lambda K T}(\lambda K T)^{i}}{i !} \\
& =1-\sum_{i=0}^{-\lambda K T} \frac{e^{i}(\lambda K T)^{i}}{i !}
\end{aligned}
$$

The number of time frames (including the current awake time frame) before the next awake time frame for an MSS is calculated as the smallest value of $K$ such that $P_{\text {Acc }}(K$, Data_TH $)$ is higher than a predefined probability threshold denoted by Prob_TH. The length of one awake-and-sleep cycle, denoted by $K^{*}$, is calculated as follows:

$$
\begin{aligned}
K^{*} & =\text { LengthAwkSlpCyl }(\lambda, \text { Data_TH }) \\
& =\operatorname{Min}\left\{K \mid P_{\text {Acc }}(K, \text { Data_TH }) \geq P r o b_{-} T H\right\}
\end{aligned}
$$

Note that an awake-and-sleep cycle is composed of the current awake time frame and the following sleep window. The size of the sleep window in a cycle is therefore $K^{\prime \prime}-1$, which is sent by the BS to the currently awake MSSs for entering the sleep mode.

Given the threshold of data accumulation, the best case for an MSS in terms of power saving is to make the MSS a single-member group resulting in the largest value of $K^{\prime \prime}$. Therefore, instead of treating all MSSs as one group as in LBPS-Aggr, we could firstly make each MSS a singlemember group for $K^{\prime \prime}$ calculation. Since the load of each 


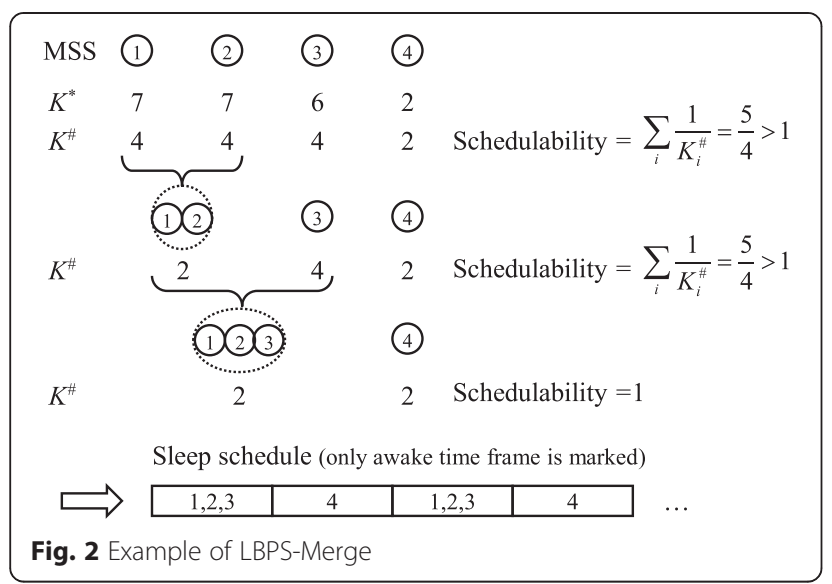

MSS varies, each group usually has a different value of $K^{*}$. In order to achieve a better gain of power saving, the sleep scheduling algorithm should be able to accommodate different values of $K^{\prime \prime}$ as long as a feasible sleep schedule can be found. In the case that a feasible sleep schedule cannot be found for the current state of grouping, merging of some groups is necessary. This idea of treating each MSS as a single-member group from the start and merging groups when necessary leads to an enhanced protocol, namely LBPS-Merge.

Since it is difficult to check the schedulability of groups with any possible value of $K^{*}$, the value of $K^{\prime \prime}$ is converted to the closest and smaller power of 2, denoted by $K^{\#}$ (i.e., $K=2^{\left\lfloor\log _{2} K^{*}\right\rfloor}$ ) in LBPS-Merge. With the property of powers of 2 , a quick check for schedulability can be obtained. Schedulability of a number of groups with different $K^{\#}$ values is defined by the following equation:

$$
\text { Schedulability }=\sum_{i} \frac{1}{K_{i}^{\#}}
$$

Schedulability equal to or smaller than 1 indicates that a feasible schedule can be found. Schedulability larger than 1 indicates the necessity of merging some groups. The worst case in LBPS-Merge is all MSSs be merged as one group (the same result as in LBPS-Aggr) and $K^{\#}=1$ (no sleep window). An example of the LBPS-Merge protocol for illustration purpose is displayed in Fig. 2.

\section{LTE LBPS schemes}

\subsection{Basic idea}

The previous works of LBPS-Aggr and LBPS-Merge were designed for IEEE 802.16. In LTE, the estimation of the traffic load is performed by eNB, and each UE is notified of the length of the DRX cycle by eNB via RRC signaling as illustrated in Fig. 3. Moreover, applying the LBPS schemes to LTE requires proper addressing of the features of LTE as discussed in the following:

(1)As illustrated in Fig. 4, the basic time unit for packet scheduling and transmission in LTE is called a TTI (transmission time interval) with a length of $1 \mathrm{~ms}$. Thus, TTI is the time unit for LBPS to estimate the length of the sleep cycle in LTE. In each TTI, a scheduling decision is made where each scheduled UE is assigned a certain amount of radio resources in the

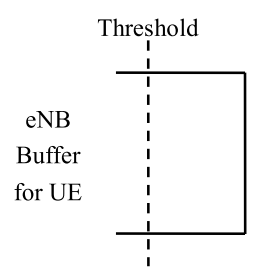

eNB estimates next LBPS Cycle length \& Notifies UE

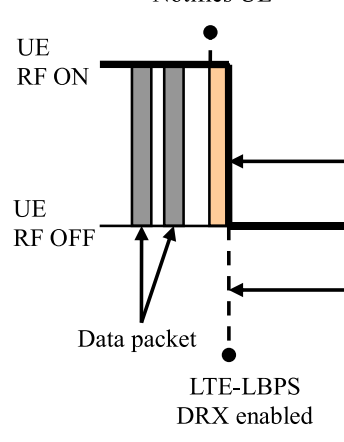

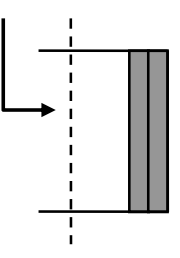
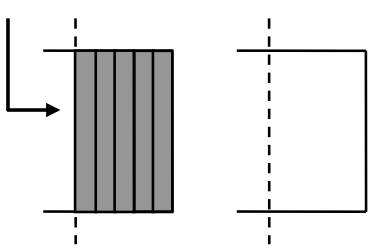

eNB estimates next LBPS Cycle length \& Notifies UE

Fig. 3 Illustration of LTE LBPS 


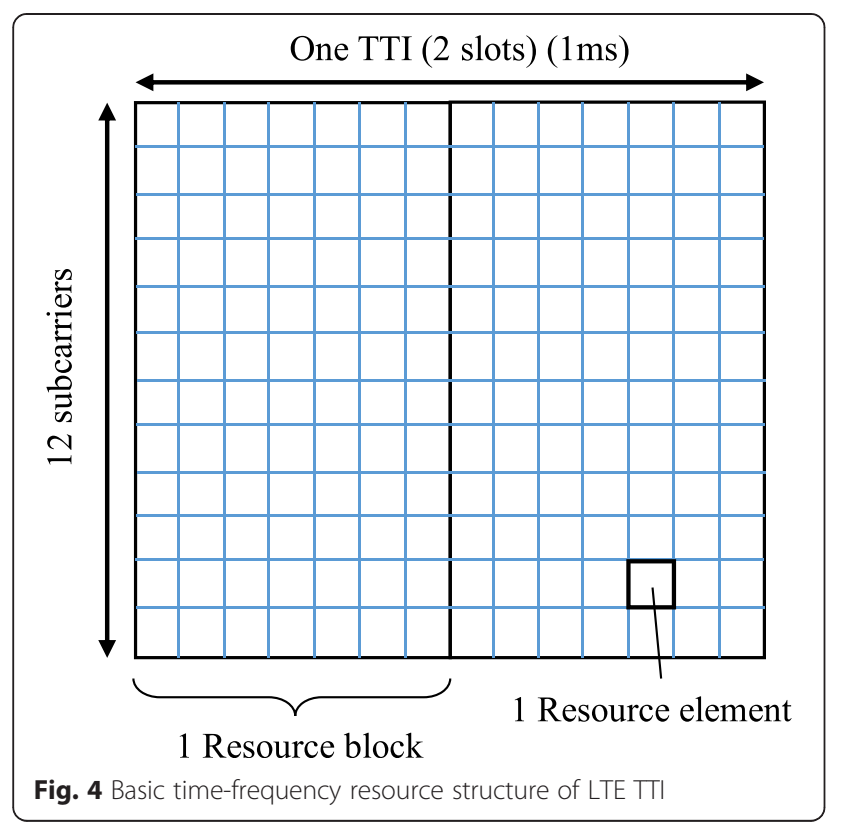

time and frequency domain. In the time domain, a TTI is split into two 0.5-ms slots. Each slot comprises seven orthogonal frequency-division multiplexing (OFDM) symbols in the case of the normal cyclic prefix length. In the frequency domain, resources are grouped in units of 12 subcarriers, such that one unit of 12 subcarriers for a duration of one slot is called a resource block (RB), which is the smallest element of resource allocation. The smallest unit of resource is the resource element $(\mathrm{RE})$, which consists of one subcarrier for a duration of one OFDM symbol. Therefore, an RB is comprised of $84(7 \times 12)$ REs in the case of the normal cyclic prefix length.

(2)In our previous work in IEEE 802.16, the channel capacity was assumed to be static, and the threshold of data accumulation (DATA_TH) was practically set as the size of a time frame (i.e., the number of bits that can be carried in a time frame). In LTE, the channel capacity varies due to the adaptively selected Modulation and Coding Scheme (MCS) for each UE. LTE eNB typically selects MCS for a UE depending on a prediction of the DL channel condition, which is according to the channel quality indicator (CQI) feedback transmitted by that UE. 3GPP gives a table of reference for efficiency of each CQI index as shown in Table 1. The estimation of the channel capacity of LTE is therefore based on the CQI report from the UE and the corresponding efficiency value in Table 1. Moreover, since LTE physical control channels (such as PDCCH, PCFICH, and PHICH) also make use of the REs in the TTI, estimation of the capacity for the user data should exclude the REs reserved for the control channels.

An overview of the proposed LBPS schemes for LTE is illustrated in Fig. 5, in which estimation of the traffic load is the same as in our previous work. Capacity estimation in a TTI is presented in the next section followed by the two revised LBPS schemes for LTE, namely LTE-LBPS-Aggr and LTE-LBPS-Merge.

\subsection{Capacity estimation of TTI}

Estimation of the channel capacity depends on the CQI reports from a UE, meaning that different UEs would have different views of the channel capacity. Estimation of the channel capacity for a UE also requires to address the type of CQI report. Two types of CQI reports are addressed in this paper: Wideband report and Full-Subband report. In Wideband report, the UE reports one wideband CQI value for the whole system bandwidth. In Full-Sub-band report, in addition to the wideband CQI value, the UE reports a CQI value for each sub-band with system-defined sub-band size. Notations used in estimation of the channel capacity for $\mathrm{UE}_{i}$ are defined as follows and also illustrated in Fig. 6.

$N_{\text {OFDM }}^{\text {TTI }}$ The number of OFDM symbols (REs) in a TTI, which is 14 in the case of the normal cyclic prefix length $N_{\text {OFDM }}^{\text {Ctrl }}$ The number of OFDM symbols used by the control channels in a TTI

$N_{\text {OFDM }}^{\text {Resv }}$ The number of OFDM symbols reserved for reference signals in a TTI of 12 subcarriers

Table $1 \mathrm{CQ}$ table by 3GPP

\begin{tabular}{llll}
\hline CQI index & Modulation & Approximate code rate & Efficiency (bits/RE) \\
\hline 0 & No Tx & - & - \\
1 & QPSK & 0.076 & 0.1523 \\
2 & QPSK & 0.12 & 0.2344 \\
3 & QPSK & 0.19 & 0.3770 \\
4 & QPSK & 0.3 & 0.6016 \\
5 & QPSK & 0.44 & 0.8770 \\
6 & QPSK & 0.59 & 1.1758 \\
7 & 16QAM & 0.37 & 1.4766 \\
8 & 16QAM & 0.48 & 1.9141 \\
9 & 16QAM & 0.6 & 2.4063 \\
10 & 64QAM & 0.45 & 2.7305 \\
11 & 64QAM & 0.55 & 3.3223 \\
12 & 64QAM & 0.65 & 3.9023 \\
13 & 64QAM & 0.75 & 4.5234 \\
14 & 64QAM & 0.85 & 5.1152 \\
15 & 64QAM & 0.93 & 5.5547 \\
\hline
\end{tabular}




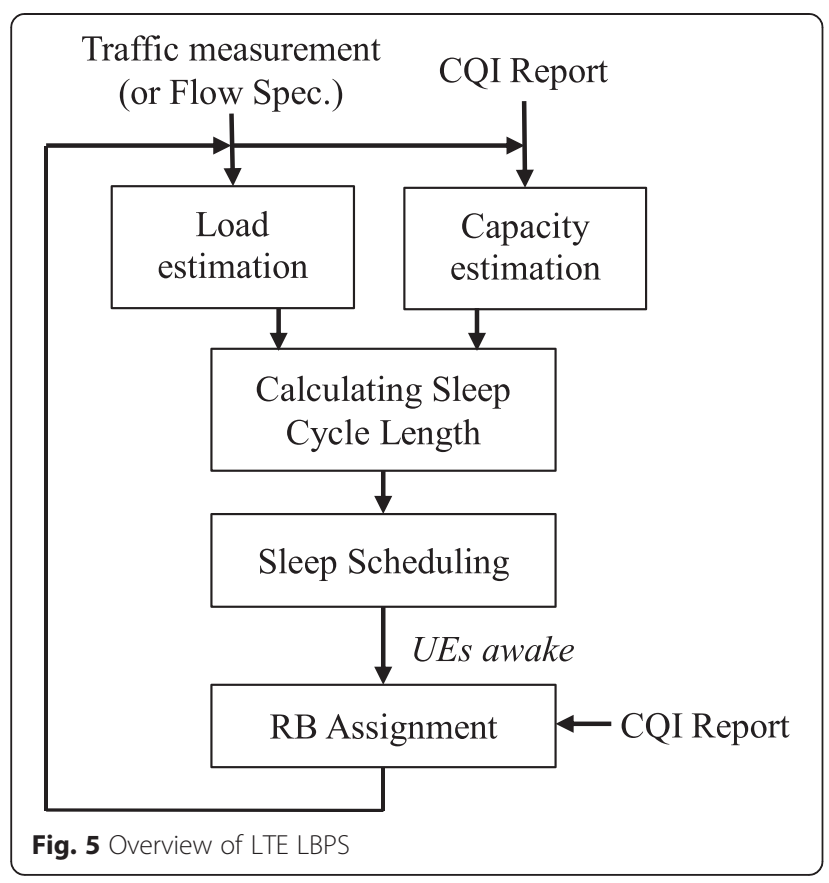

Note that the two RBs in a TTI of 12 subcarriers are called a resource block group (RBG) in the paper. Therefore, the number of REs for the user data in an RBG, denoted by $N_{\mathrm{RE}}^{\mathrm{TTI}}$, is calculated as follows:

$$
N_{\mathrm{RE}}^{\mathrm{TTI}}=\left(N_{\mathrm{OFDM}}^{\mathrm{TTI}}-N_{\mathrm{OFDM}}^{\mathrm{Ctrl}}\right) \times 12(\text { subcarriers })-N_{\mathrm{OFDM}}^{\mathrm{Resv}}
$$

For the case of Wideband report, the channel capacity estimated for $\mathrm{UE}_{i}$ in a TTI, denoted by $C_{i}^{W}$, is calculated as follows:

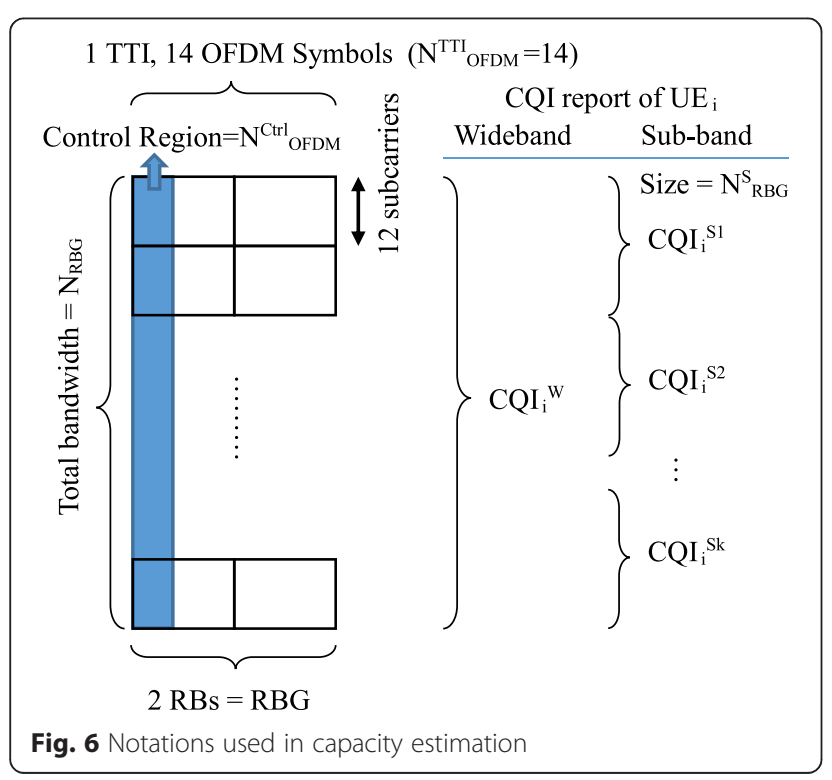

$$
C_{i}^{W}=N_{\mathrm{RE}}^{\mathrm{TTI}} \times \operatorname{Eff}\left(\mathrm{CQI}_{i}^{W}\right) \times N_{\mathrm{RBG}}
$$

The function of Eff $\left(\mathrm{CQI}_{i}^{W}\right)$ in Eq. 6 returns the efficiency value for the given wideband CQI value $\mathrm{CQI}_{i}^{W}$ according to Table 1 , and $N_{\text {RBG }}$ is the total number of RBG in the system.

For the case of Full-Sub-band report, the channel capacity estimated for $\mathrm{UE}_{i}$ in a TTI, denoted by $C_{i}^{S}$, is calculated as follows:

$$
C_{i}^{S}=\sum_{\forall S_{k}}\left(N_{\mathrm{RE}}^{\mathrm{TTI}} \times \operatorname{Eff}\left(\mathrm{CQI}_{i}^{S_{k}}\right) \times N_{\mathrm{RBG}}^{S}\right)
$$

Note that $\mathrm{CQI}_{i}^{S_{k}}$ is the CQI value for sub-band $S_{k}$, and $N_{\mathrm{RBG}}^{S}$ is the number of RBG in a sub-band.

As in the estimation of the traffic load for a UE, the estimation of the current channel capacity for $\mathrm{UE}_{i}$, denoted by $C_{i}$, is calculated by exponentially averaging the samples of each calculation. The channel capacity for all UEs is calculated by combining the channel capacity estimated by each individual UE with the ratio of the UE's traffic load in the group. The channel capacity (bits/TTI) for all UEs, denoted by $C_{\text {Channel, }}$, is calculated as follows:

$$
C_{\text {Channel }}=\sum_{\forall U E_{i}}\left(C_{i} \frac{\lambda_{i}}{\lambda}\right)
$$

where $\lambda$ is the total DL load and $\lambda_{i}$ is the current load of $\mathrm{UE}_{i}$

\subsection{LTE-LBPS-Aggr and LTE-LBPS-Merge}

As presented in Section 2.3, all UEs are grouped together in sleep scheduling and all of the traffic flows are treated as an aggregate flow in the scheme of LTELBPS-Aggr. Therefore, the length of the next LBPS DRX cycle (denoted by $K_{\text {Aggr }}^{*}$ in units of TTI) is calculated according to Eqs. 3 and 8 as follows:

$$
\begin{aligned}
K_{\text {Aggr }}^{*}= & \text { LengthAwkSlpCyl }(\lambda, \text { Data_TH }), \\
& \text { where Data_TH }=\alpha \cdot C_{\text {Channel }}
\end{aligned}
$$

Note that the threshold of data accumulation in LTELBPS-Aggr is set as a percentage $(\alpha)$ of the capacity for the user data in a TTI in order to reduce the probability of buffer overflow (e.g., $\alpha=0.8$ in the simulation).

The scheme of LTE-LBPS-Merge adopts a different strategy for sleep scheduling in which each UE is initially treated as a single-member group and a series of merging operations is performed until a feasible sleep schedule is found. The difference of LTE-LBPS-Merge over the previous work of LBPS-Merge includes the following: (1) the value of Data_TH in calculating the sleep cycle length for each group is based on the load of the group as well as the estimated channel capacity as presented in Section 3.2, and (2) in order to take advantage of multi-user diversity 
Table 2 Simulation parameters

\begin{tabular}{|c|c|c|c|}
\hline \multicolumn{2}{|c|}{ System parameters } & \multicolumn{2}{|c|}{ Contrast schemes } \\
\hline \multirow[t]{5}{*}{ Channel related } & $N_{\mathrm{OFDM}}^{\Pi 1}=14$ & Std. DRX1 & On duration $=1 \mathrm{~ms}$ \\
\hline & $N_{\text {OFDM }}^{\mathrm{Ctrl}}=2$ & & Inactivity timer $=10 \mathrm{~ms}$ \\
\hline & $N_{\text {OFDM }}^{\text {Resv }}=0$ & & Short DRX Cycle $=80 \mathrm{~ms}$ \\
\hline & $N_{\mathrm{RBG}}=100$ & & Short Cycle Timer $=2$ \\
\hline & $N_{\mathrm{RBG}}^{S}=8$ & & Long DRX Cycle $=320 \mathrm{~ms}$ \\
\hline \# UE & 12, 60 (equal load) & Std. DRX2 & On duration $=1 \mathrm{~ms}$ \\
\hline \multirow[t]{3}{*}{ Type of UE } & H-type: CQI 11 15 & & Inactivity timer $=10 \mathrm{~ms}$ \\
\hline & M-type: CQI 6 10 & & Short DRX Cycle $=160 \mathrm{~ms}$ \\
\hline & L-type: CQI 1 5 & & Short Cycle Timer $=2$ \\
\hline CQI Reporting & 5 ms (periodically) & & Long DRX Cycle $=640 \mathrm{~ms}$ \\
\hline Packet size & 799 bits, 3995 bits & M-ADRX & On duration: $1 \mathrm{~ms}$ \\
\hline \multicolumn{2}{|c|}{ LTE-LBPS-Aggr, LTE-LBPS-Merge } & & Inactivity timer: adaptive \\
\hline DATA_TH & $C_{\text {Channel }} \times a, a=0.8$ & & Short DRX Cycle: 160 ms \\
\hline Prob_TH & 0.8 & & DRX Short Cycle Timer:2 \\
\hline MinGroupSize & 2 & & Long DRX Cycle: 640 ms \\
\hline
\end{tabular}

in resource allocation, the constraint of minimum group size (denoted by MinGroupSize, set as 2 in the simulation) is added into LTE-LBPS-Merge. Major steps in LTELBPS-Merge are presented as follows:

Step 1: Estimate the current load of each UE, and each UE initially forms a group.

Step 2: Sort the groups in the ascendant order of load. For each group, calculate $K_{G}$ " $=$ LengthAwkSlpCyl $\left(\lambda_{G}\right.$, Data_TH $)$, where $\lambda_{G}=$ the total load in a group and Data_TH $=\alpha \cdot C_{\text {Channel }}$ (Note that $C_{\text {Channel }}$ is calculated according to Eq. 8 considering only the UEs in the group). Convert each group's $K_{G}^{*}$ to the closest and smaller power of 2, i.e., $K_{G}^{\#}=2\left\lfloor^{\left.\log _{2} K_{G}^{*}\right\rfloor}\right.$.

Step 3: (Schedulability and MinGroupSize Check) If (Schedulability $=\sum \frac{1}{K_{G}^{\#}} \leq 1$ ) and (each group's size $\geq$ MinGroupSize)

The algorithm stops and returns the final sleep schedule.

\section{Else (merging operation)}

Try to merge the smallest-load group with another group until a non-degraded merge is found, or merge the two groups with the smallest loads (degraded merge).

Repeat step 2 and step 3.

(Note that merging of two groups that does not result in a smaller value of $K^{\#}$ is called a non-degraded merge. A degraded merge is performed only when a non-degraded merge cannot be found. For details, please refer to our previous work [17].)

\section{Performance evaluation}

\subsection{Simulation environment and metrics}

A simulation study is conducted to evaluate the performance of the proposed LTE-LBPS-Aggr and LTELBPS-Merge as well as some contrast schemes. A custom program developed by Microsoft Visual $\mathrm{C}++$ for system level simulation is extended from the authors' previous tool in IEEE 802.16e. The input load for each UE is equal and the packet arrival process is Poisson. The size of a packet is fixed and two levels of packet size, 799 and 3996 bits, are simulated. The channel quality for each UE is simulated by directly drawing a random number from the range of CQI values. The number of bits that can be carried in a resource block is calculated according to the efficiency of the CQI value.

In order to simulate different cases of the channel quality, three types of UEs are defined. An H-type (high link quality) UE is assumed to use 64QAM modulation with CQI value ranging from 10 to 15 . An $M$-type (medium link quality) UE uses 16QAM modulation with CQI value ranging from 7 to 9. An L-type (low link quality) UE uses QPSK modulation with CQI value ranging from 1 to 6 . In the simulation, the total number of RBG $\left(N_{\mathrm{RBG}}\right)$ is 100 (for a $20-\mathrm{MHz}$ bandwidth) and the average channel capacity of an H-type UE is $56.19 \mathrm{Mbps}$ for Wideband report and 58.38 Mbps for Full-Sub-band report. M-type is 24.41 Mbps (Wideband report) and 26.86 Mbps (Full-Sub-band report). L-type is $5.43 \mathrm{Mbps}$ (Wideband report) and 6.22 Mbps (Full-Sub-band report). The total input load (denoted by $\lambda$ Mbps) in the simulation depends on the type of UEs in the network, and the utilization factors $\rho_{\mathrm{WB}}$ and $\rho_{\mathrm{SB}}$ are defined 
Table $\mathbf{3}$ Inactivity timer adjustment in M-ADRX

\begin{tabular}{lll}
\hline SINR & Modulation & Inactivity timer \\
\hline $11.738 \sim+\infty$ & 16QAM & $4 \mathrm{~ms}$ \\
$10.889 \sim 11.738$ & 16QAM & $5 \mathrm{~ms}$ \\
$9.2851 \sim 10.889$ & 16QAM & $10 \mathrm{~ms}$ \\
$6.509 \sim 9.2851$ & 16QAM & $20 \mathrm{~ms}$ \\
$5.4524 \sim 6.509$ & 16QAM & $30 \mathrm{~ms}$ \\
$4.7423 \sim 5.4524$ & QPSK & $40 \mathrm{~ms}$ \\
$3.6543 \sim 4.7432$ & QPSK & $50 \mathrm{~ms}$ \\
$3.4625 \sim 3.6543$ & QPSK & $60 \mathrm{~ms}$ \\
$1.295 \sim 3.4626$ & QPSK & $80 \mathrm{~ms}$ \\
$-1.1467 \sim 1.295$ & QPSK & $100 \mathrm{~ms}$ \\
\hline
\end{tabular}

as the ratio of the total input load over the average channel capacity for the case of Wideband report (denoted by WB) and Full-Sub-band report (denoted by SB), respectively.

Two standard DRX operations (denoted by Std. DRX1 and Std. DRX2), each with different DRX parameter settings, are simulated for the purpose of performance comparison. The scheme of M-ADRX [30] in which the inactivity timer is adjusted based on the signal-to-interference-plus-noise-ratio (SINR) value is also included as one of the contrast schemes. Simulation parameters of the network system, proposed schemes, and contrast schemes are listed in Table 2. Conditions for the SINRbased adjustment of the inactivity timer in M-ADRX in the simulation are displayed in Table 3. Note that all of the simulated schemes adopt the strategy of maximum throughput [35] for RB assignment, in which each RB is allocated to the awake UE that can achieve the maximum throughput in the current TTI.

Performance criteria investigated in the simulation study include power saving efficiency (denoted by PSE), average

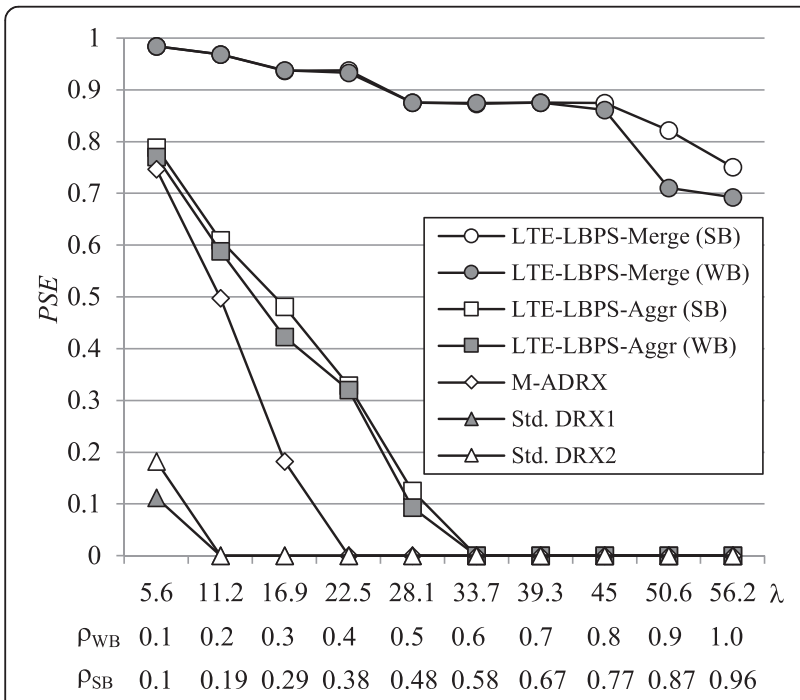

Fig. 7 PSE in the case of all-H UES

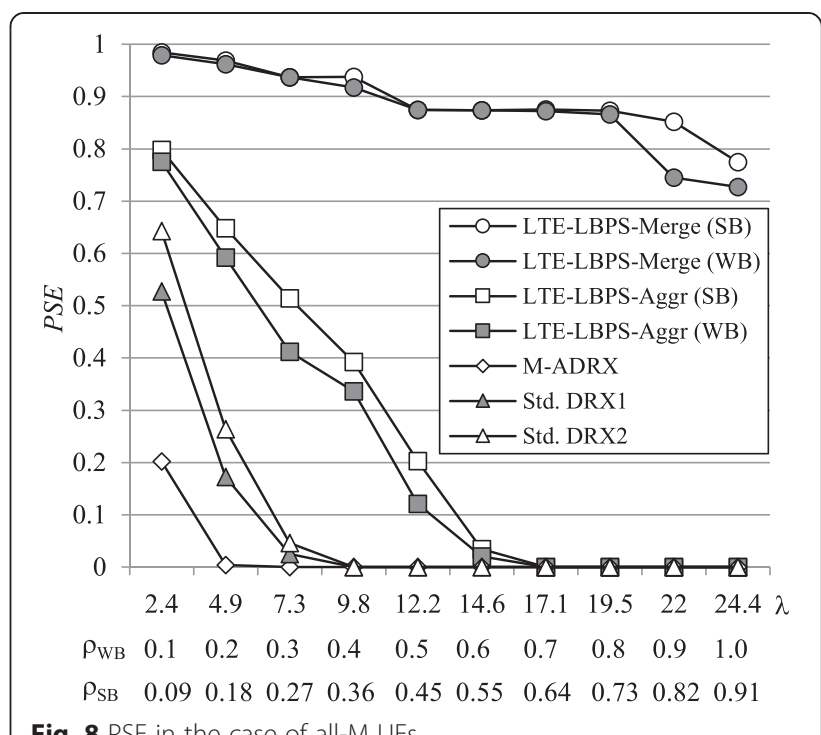

delay (denoted by $A v g D$ ), and signaling overheads for DRX operation (denoted by SignalDRX) and CQI reporting (SignalCQI), respectively. PSE is defined as the ratio of radio-off time. SignalDRX is defined as the rate of signaling messages ( $R R C$ connection reconfiguration) for configuring DRX parameters. SignalCQI is defined as the rate of signaling messages for sending CQI reports.

\subsection{Simulation results}

\subsubsection{PSE and delay}

Figures 7, 8, and 9 show the result of PSE for each of the following three input cases: all-H-type UEs, all-M-type UEs, and all-L-type UEs. In the figures, there are three rows of index for the $x$-axis. The upper row is the input load $\lambda$ in

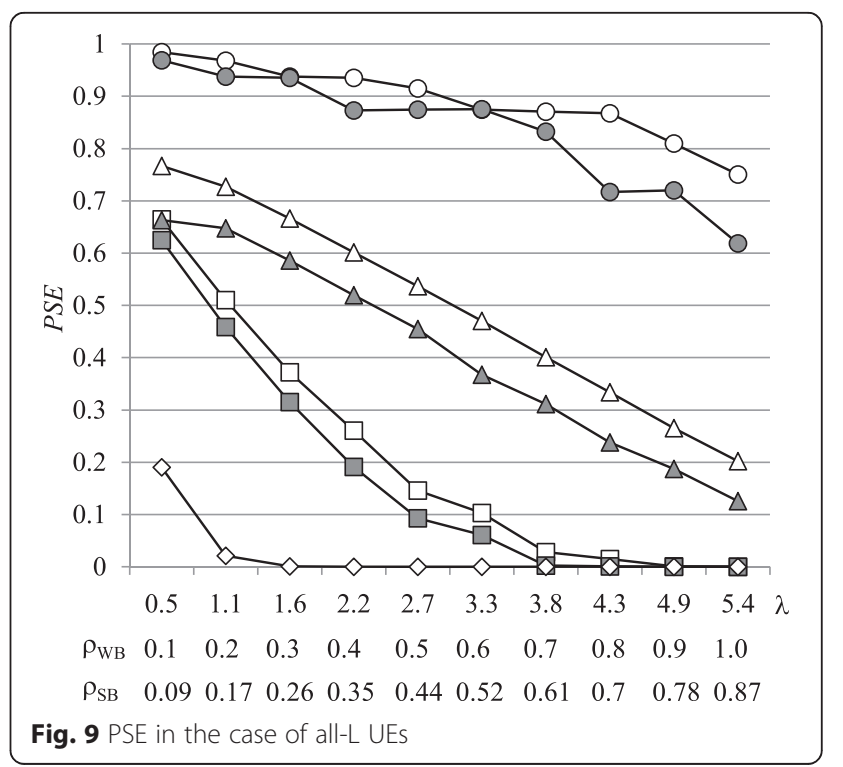




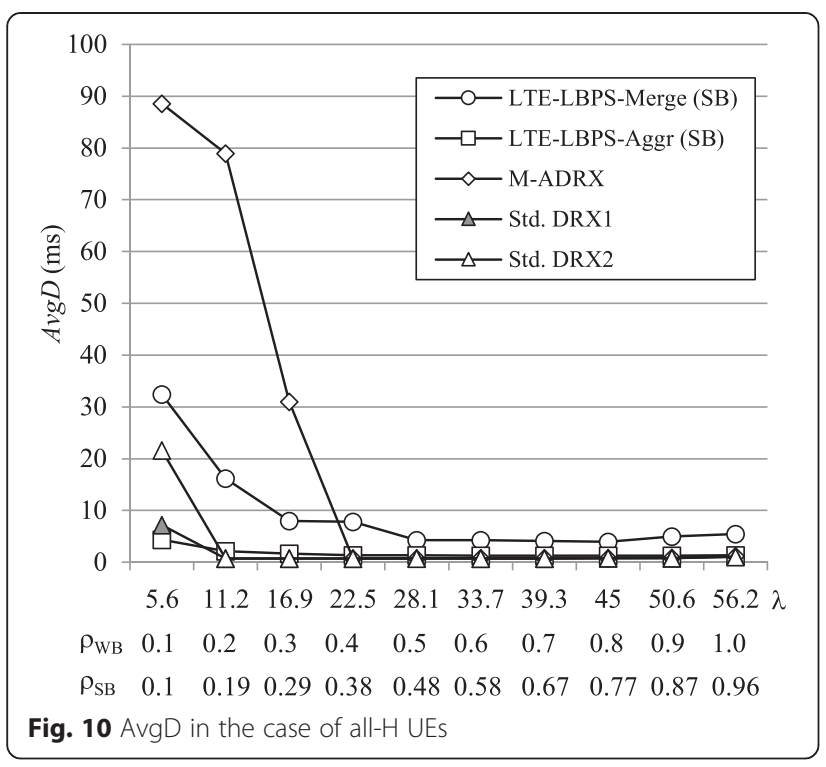

Mbps, the middle row is the utilization factor $\rho_{\mathrm{WB}}$ for the case of Wideband report (WB), and the lower row is the utilization factor $\rho_{\mathrm{SB}}$ for the case of Full-Sub-band report (SB). The corresponding result of AvgD for each UE case is displayed in Figs. 10, 11, and 12, respectively. Note that the delay performance of the proposed schemes for the case of WB is pretty close to that for the case of SB in Figs. 10, 11, and 12; therefore, only the curve of SB is displayed for compactness. The following observations can be made from Figs. 7, 8, 9, 10, 11, and 12:

(1) The scheme of LTE-LBPS-Merge (in both WB and SB) outperforms that of LTE-LBPS-Aggr and the other schemes in terms of PSE in all UE cases. The reason for the better PSE of LTE-LBPS-Merge than that of

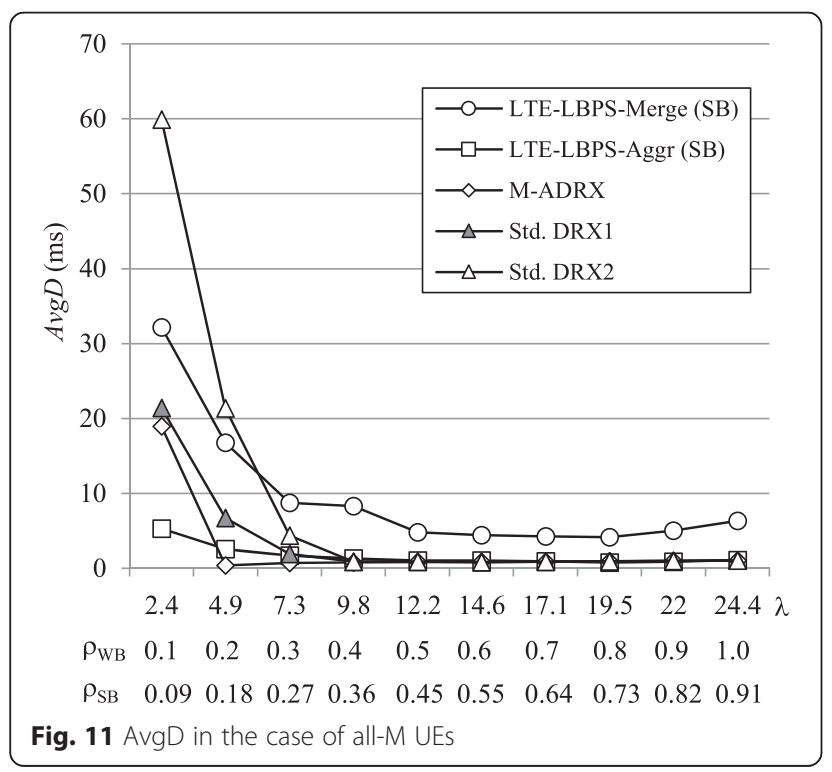

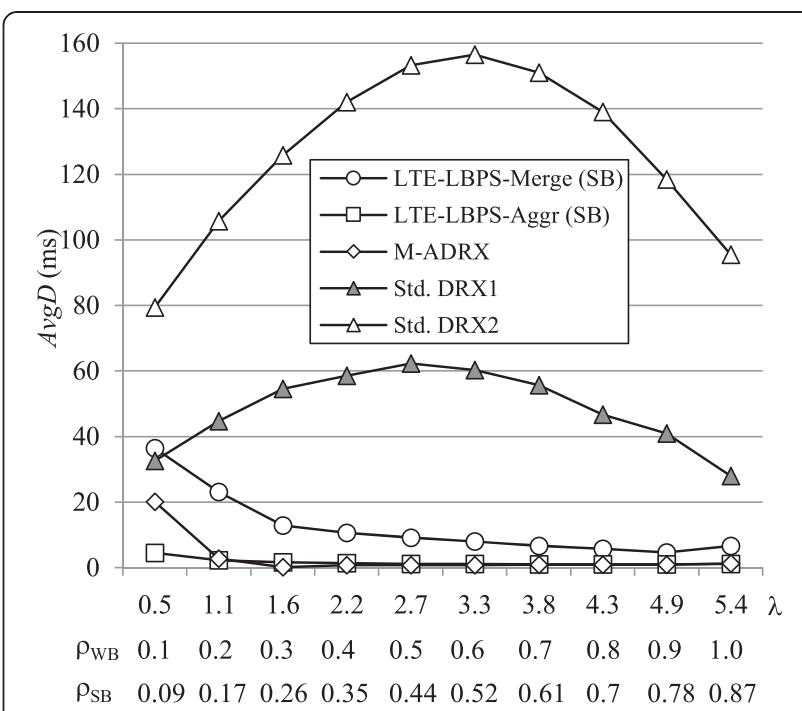

Fig. $12 \mathrm{AvgD}$ in the case of all-L UES

LTE-LBPS-Aggr is LTE-LBPS-Merge's flexibility in sleep scheduling. However, LTE-LBPS-Merge achieves higher PSE at the cost of longer AvgD in comparison with LTE-LBPS-Aggr.

(2)Moreover, LTE-LBPS-Merge achieves higher PSE than the contrast schemes (Std. DRX1, Std. DRX2, and M-ADRX) no matter what the input load is. The reason is threefold. Firstly, UEs in LTE-LBPSMerge enter the sleep mode earlier than in the contrast schemes since there is no inactivity timer period in the LBPS scheme. Secondly, data arrival in the contrast schemes immediately makes UEs go back to the active mode, while LTE-LBPS-Merge retains the sleep mode of UEs until the pre-calculated awake time is reached. Lastly, grouping UEs in sleep scheduling in LTE-LBPS-Merge lengthens

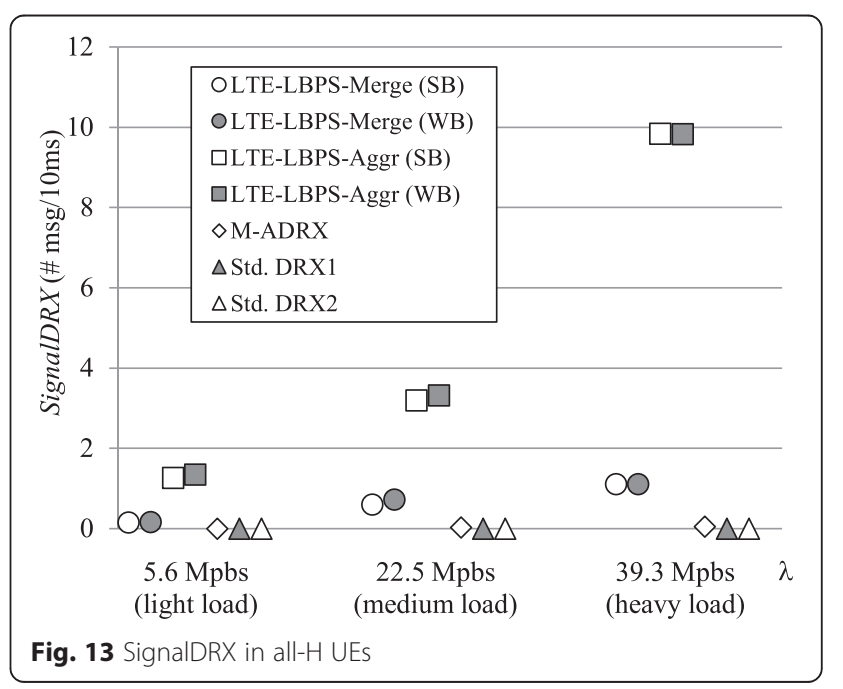




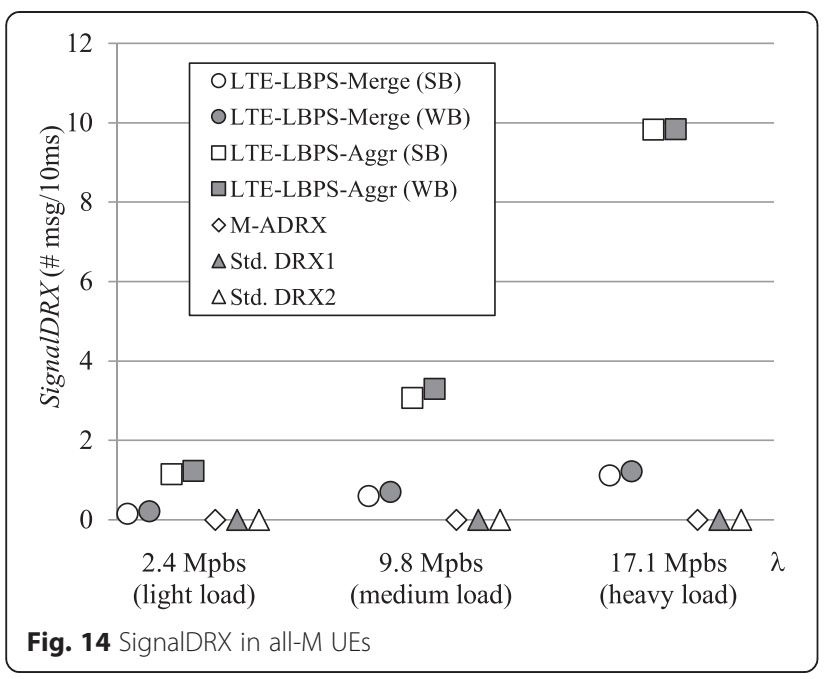

the sleep cycle length of UEs, resulting a much higher PSE.

(3) The value of PSE goes down as the input load increases in all schemes, but the PSE curves of LTE-LBPS-Merge do not drop as sharply as those of the other schemes. The reason is LTE-LBPS-Merge determines the sleep cycle length based on the estimation of data accumulation instead of passively getting back to the active mode for each packet arrival as the contrast schemes do. Furthermore, compared with LTE-LBPS-Aggr, multiple groups of UEs in sleep scheduling in LTE-LBPS-Merge make a lower load for each group; therefore, the impact of the input load on PSE is reduced.

(4)LTE-LBPS-Aggr outperforms Std. DRX1 and Std. DRX2 in terms of PSE and AvgD, except in the case of all L-type UEs in Fig. 9, since in the case of all L-type UEs, the arrival rate of packet with fixed size (799 bits) is pretty low even under high utilization, triggering the inactivity timer of Std. DRX1 and Std.

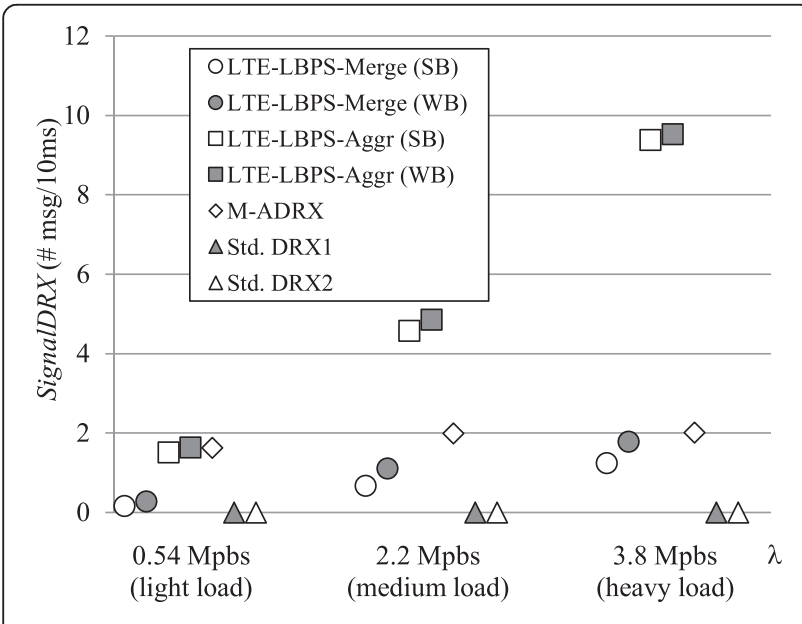

Fig. 15 SignalDRX in all-L UES

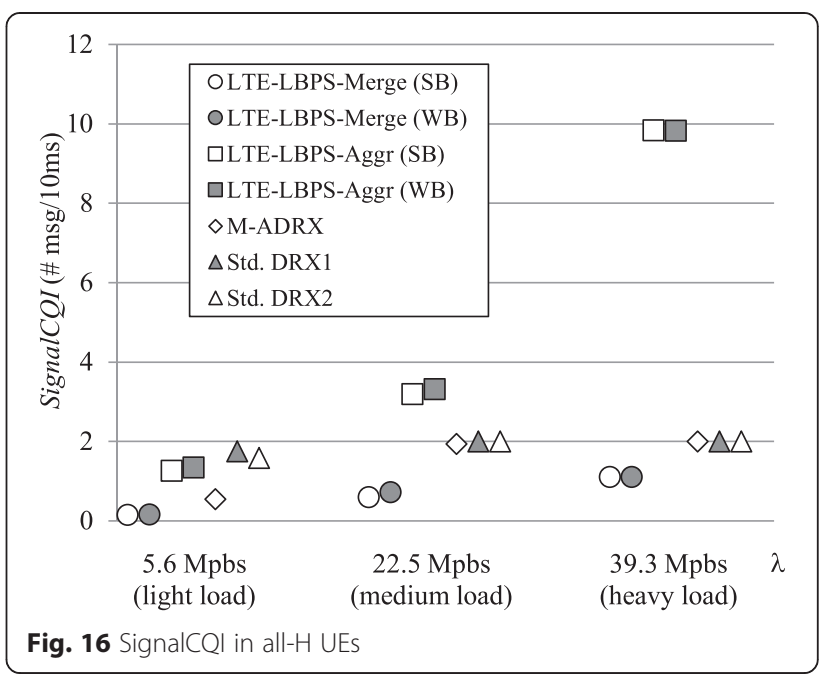

DRX2 to expire more frequently and achieve higher PSE at the cost of unacceptable high AvgD in Fig. 12.

(5) PSE of SB is slightly higher than that of WB in all cases for LTE-LBPS-Aggr and LTE-LBPS-Merge. The reason is that the channel capacity of SB is a little bit higher than the channel capacity of WB, making the utilization factor of $\rho_{\mathrm{SB}}$ slightly smaller than that of $\rho_{\mathrm{WB}}$ for the same input load, which results in longer sleep cycle length for the case of SB.

(6) PSE of Std. DRX2 is better than that of Std. DRX1 because of the longer cycle length in Std. DRX2.

Moreover, under the same utilization, two standard DRX schemes can achieve better PSE in the case of all-L-type UEs than in the case of all-M-type UEs and all-H-type UEs, which is due to load reduction in the case of all-L-type UEs resulting in less packet arrival rate and more frequent expiration of the inactivity timer.

(7) The contrast scheme of M-ADRX performs better in terms of PSE in the case of all-H-type UEs than in

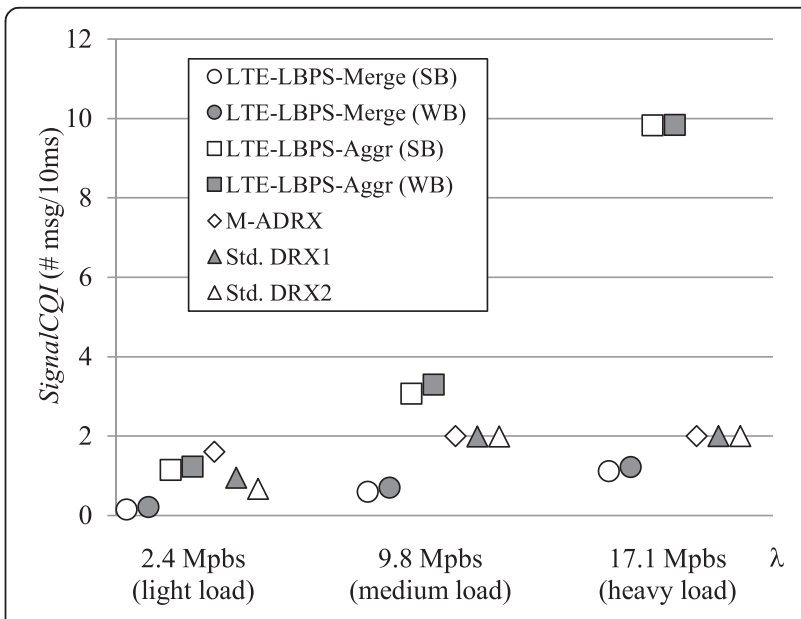

Fig. 17 SignalCQI in all-M UES 


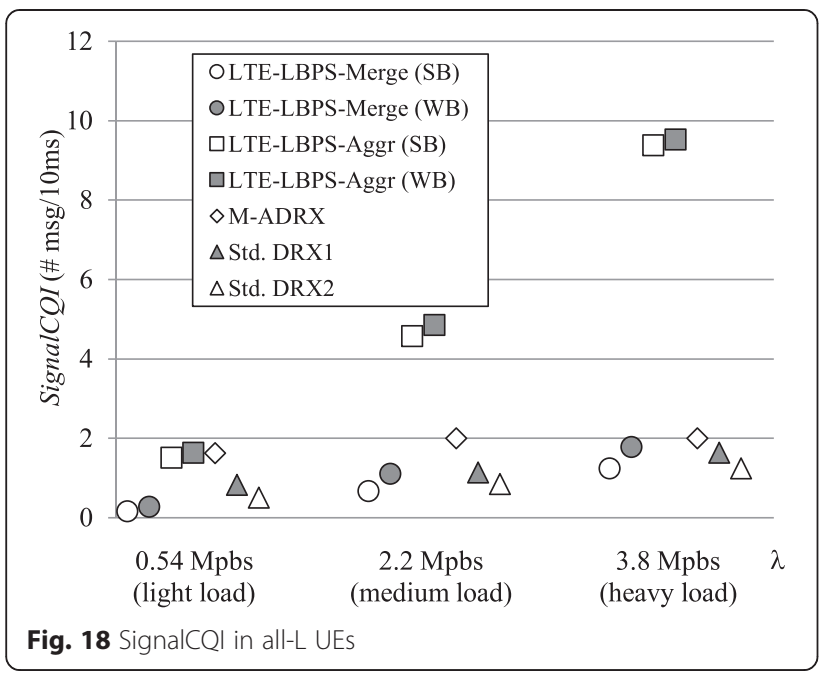

other UE cases, since as shown in Table 3, M-ADRX selects a shorter inactivity timer for better SINR (generally better CQI value) making it easier to enter the DRX cycle in the case of all-H-type UEs.

(8) The behavior of AvgD with the increase of the input load for all schemes in Figs. 10, 11, and 12 is different from what we usually expect according to the queuing theory. The reason is that the network system with power saving is non-work-conserving, and the average delay is affected by not only the input load but also the sleeping mechanism. More specifically, both the increase of load and the increase of PSE contribute to the increase of AvgD. However, load increasing makes lower PSE. Therefore, as the load increases, the curve of AvgD will either go up or go down, depending on which factor makes a higher impact on the average delay.

In summary, the figures have demonstrated that the proposed schemes of LTE-LBPS-Merge and LTE-LBPSAggr can adapt to different input loads as well as channel capacities and outperform the contrast schemes in terms of PSE at the cost of a moderate increase in AvgD.

\subsubsection{Signaling overhead}

Results of SignalDRX are displayed in Figs. 13, 14, and 15. Results of SignalCQI are displayed in Figs. 16, 17, and 18. The following observations can be made from the figures:

(1)SignalDRX of LTE-LBPS-Aggr is larger than that of LTE-LBPS-Merge in all cases since the smaller sleep cycle length by LTE-LBPS-Aggr results in more frequent notification of the next cycle length. The same reason is also applied to the higher SignalCQI of LTE-LBPS-Aggr than of LTE-LBPS-Merge in CQI reporting. Moreover, as the input load increases, SignalDRX and SignalCQI of both LBPS schemes increase due to the decreased sleep cycle length.

(2) Since DRX configuration for Std. DRX1 and Std. DRX2 is fixed after initialization, SignalDRX values of the two DRX schemes are both zero. On the other hand, M-ADRX adjusts the value of the inactivity timer according to link quality; however, for the same type of UEs (e.g., M-type), the link quality does not change a lot, making the value of the inactivity timer remain the same for most of the time and resulting in smaller values of SignalDRX for M-ADRX.

(3) The value of SignalCQI for Std. DRX1, Std. DRX2, and M-ADRX increases as the load increases, since under heavier load the chance for the three schemes entering the DRX mode is getting lower, which results in more CQI reporting for packet scheduling.

In summary, although LTE-LBPS-Aggr generates a relatively high signaling overhead in both SignalDRX and SignalCQI, the signaling overhead of LTE-LBPS-Merge is moderate in comparison with the contrast schemes.

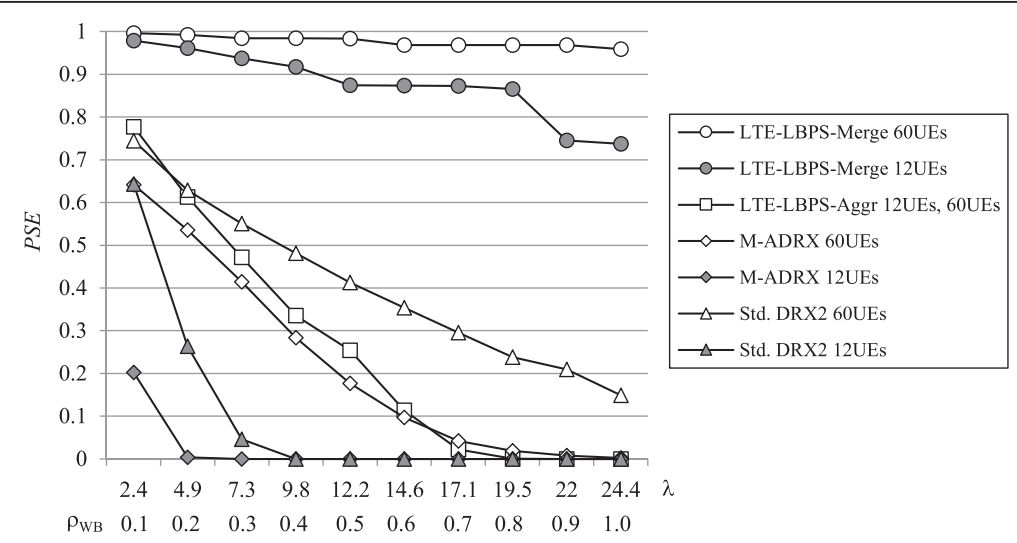

Fig. 19 Impact of number of UES on PSE (all-M UES, WB) 


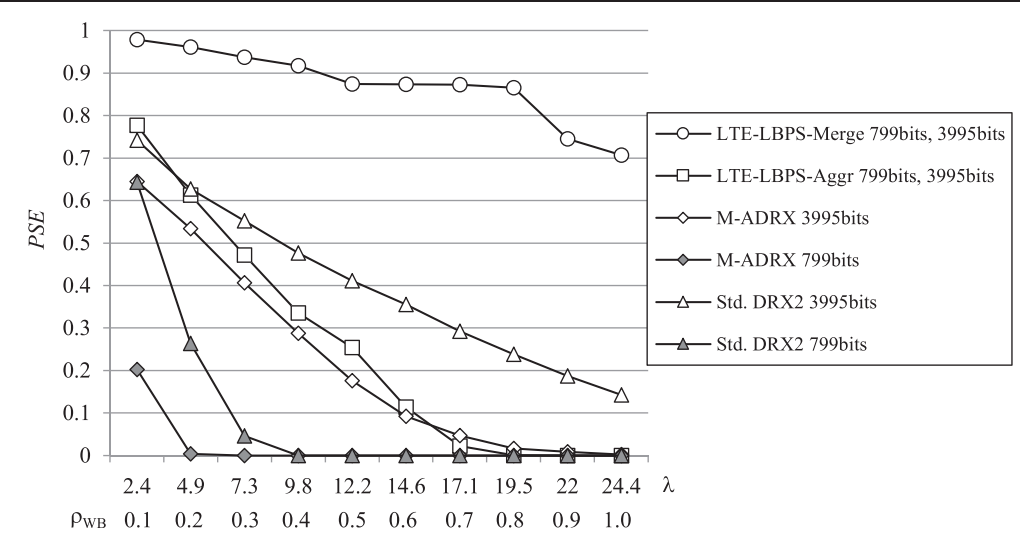

Fig. 20 Impact of packet size on PSE (all-M UEs, WB)

\subsubsection{Impact of the number of UEs and packet size}

Figure 19 shows the PSE result of each scheme under 12 UEs as well as 60 UEs for investigating the impact of the number of UEs on PSE. As shown in the figure, all schemes except LTE-LBPS-Aggr perform better under a larger number of UEs because of the reduced load for each UE for the same total input load. Moreover, the increase of the number of UEs increases the flexibility of grouping UEs in LTELBPS-Merge, which creates more benefit in terms of PSE.

Figure 20 shows the PSE result of each scheme under different packet sizes, in which a larger packet size results in higher PSE for the contrast schemes of Std. DRX2 and MADRX. The reason is that a larger packet size for the same input load makes the packet arrival time more sparsely distributed, which makes the UEs in the contrast schemes enter the sleep mode more frequently. Changes in the packet size do not affect the value of PSE of LTE-LBPSAggr and LTE-LBPS-Merge, since the sleep cycle length in both schemes is calculated based on the accumulated amount of data in bits instead of the number of packets.

\section{Conclusions}

Based on our previously proposed Load-Based Power Saving (LBPS) in the IEEE 802.16 network, two revised schemes, namely LTE-LBPS-Aggr and LTE-LBPS-Merge, are proposed for the LTE network in this paper. The proposed schemes make use of load estimation and channel capacity estimation to calculate the length of the sleep cycle in LTE DRX mode. LTE-LBPS-Aggr treats all UEs under the same eNB as a group in sleep scheduling, while LTE-LBPS-Merge allows multiple groups of UEs each with different cycle lengths. The mechanism of channel capacity estimation based on CQI reporting and the algorithms of the proposed schemes are presented. The simulation study demonstrated that LTE-LBPS-Aggr can achieve high power saving efficiency, and LTE-LBPS-Merge outperforms all contrast schemes in terms of highest power saving efficiency, moderate access delay, and moderate signaling overhead. Simulation results also shows that in comparison with other schemes, the proposed scheme of LTE-LBPS-Merge can achieve a significantly higher level of power saving efficiency under heavy load. Moreover, given the same input load, the increase of the number of UEs makes power saving efficiency of LTE-LBPS-Merge even higher.

\section{Competing interests}

The authors declare that they have no competing interests.

\section{Acknowledgements}

Part of the research work in this paper has been published in Proc. of the IAENG International Conference on Communication Systems and Applications (International MultiConference of Engineers and Computer Scientists, IMECS), 12-14 March 2014, Hong Kong.

This work was supported in part by the National Science Council, Taiwan, under grant no. NSC 102-2221-E-260-013.

\section{Author details}

${ }^{1}$ Department of Computer Science and Information Engineering, National Chi Nan University, Puli, Nantou County, Taiwan. ²Department of Information Networking Technology, Hsiuping University of Science and Technology, Taichung City, Taiwan. ${ }^{3}$ Department of Sport Management, National Taiwan University of Sport, Taichung City, Taiwan.

Received: 16 April 2015 Accepted: 17 August 2015

Published online: 17 September 2015

\section{References}

1. MR Rahimi, N Venkatasubramanian, S Mehrotra, AV Vasilakos, MAPCloud: mobile applications on an elastic and scalable 2-tier cloud architecture. Proceedings, IEEE/ACM International Conference on Utility and Cloud Computing (UCC), Chicago, IL, 5-8 Nov. 2012, pp. 83-90

2. U Varshney, 4G wireless networks. IT Professional 14(5), 34-39 (2012)

3. D Pareit, B Lannoo, I Moerman, P Demeester, The history of WiMAX: a complete survey of the evolution in certification and standardization for IEEE 802.16 and WiMAX. IEEE Comm. Surv. Tutor. 14(4), 1183-1211 (2012)

4. 3GPP TS 36.300, Evolved Universal Terrestrial Radio Access (E-UTRA) and Evolved Universal Terrestrial Radio Access Network (E-UTRAN), Rel. 8, v8.5.0, (3GPP, http:// www.3gpp.org/ftp/Specs/archive/36_series/36.300/36300-850.zip, 2008)

5. 3GPP TS 36.300, Evolved Universal Terrestrial Radio Access (E-UTRA) and Evolved Universal Terrestrial Radio Access Network (E-UTRAN), Rel. 10, v10.3.0, (3GPP, http://www.3gpp.org/ftp/Specs/archive/36_series/36.300/36300-a30.zip, 2011)

6. PBF Duarte, Z Md Fadlullah, AV Vasilakos, N Kato, On the partially overlapped channel assignment on wireless mesh network backbone: a game theoretic approach. IEEE J. Sel. Area Comm. 30(1), 119-127 (2012) 
7. Y Zeng, K Xiang, D Li, AV Vasilakos, Directional routing and scheduling for green vehicular delay tolerant networks. Wirel. Netw 19(2), 161-173 (2013)

8. A Attar, H Tang, AV Vasilakos, FR Yu, VCM Leung, A survey of security challenges in cognitive radio networks: solutions and future research directions. Proc. IEEE 100(12), 3172-3186 (2012)

9. M Youssef, M Ibrahim, M Abdelatif, L Chen, AV Vasilakos, Routing metrics of cognitive radio networks: a survey. IEEE Comm. Surv. Tutor. 16(1), 92-109 (2014)

10. D López-Pérez, X Chu, AV Vasilakos, H Claussen, On distributed and coordinated resource allocation for interference mitigation in self-organizing LTE networks. IEEE/ACM Trans. Networking 21(4), 1145-1158 (2013)

11. D López-Pérez, X Chu, AV Vasilakos, H Claussen, Power minimization based resource allocation for interference mitigation in OFDMA femtocell networks. IEEE J. Sel. Area Comm. 32(2), 333-344 (2014)

12. PP Demestichas, V-AG Stavroulaki, L-MI Papadopoulou, AV Vasilakos, ME Theologou, Service configuration and traffic distribution in composite radio environments. IEEE Trans. Syst. Man. Cybern. C Appl. Rev. 34(1), 69-81 (2004)

13. MA Khan, $\mathrm{H}$ Tembine, AV Vasilakos, Game dynamics and cost of learning in heterogeneous 4G networks. IEEE J. Sel. Area Comm. 30(1), 198-213 (2012)

14. C-Y Wang, C-H Ko, H-Y Wei, AV Vasilakos, A voting-based femtocell downlink cell-breathing control mechanism. IEEE/ACM Transactions on Networking, vol. PP, no. 99, 2014

15. 3GPP Contribution, Nokia, R2-071285, DRX parameters in LTE, (Nokia, http:// www.3gpp.org/ftp/tsg_ran/WG2_RL2/TSGR2_57bis/Documents/R2-071285.zip, 2007)

16. C Bontu, E Illidge, DRX mechanism for power saving in LTE. IEEE Commun. Mag. 47(6), 48-55 (2009)

17. CC Yang, YT Mai, JY Chen, YS Shen, YC Kuo, LBPS: load-based power saving in the IEEE 802.16e network. Comput. Electr. Eng. 38(4), 891-905 (2012)

18. CC Yang, YT Mai, JY Chen, YC Kuo, Integrated load-based power saving for BS and MSS in the IEEE 802.16e network. Wirel. Commun. Mob. Comput. 13(18), 1918-1931 (2013)

19. L Zhou, H Xu, H Tian, Y Gao, L Du, L Chen, Performance analysis of power saving mechanism with adjustable DRX cycles in 3GPP LTE. Proceedings, IEEE Vehicular Technology Conference (VTC Fall), Calgary, BC, 21-24 Sept. 2008, pp. 1-5

20. S Fowler, RS Bhamber, A Mellouk, Analysis of adjustable and fixed DRX mechanism for power saving in LTE/LTE-advanced. Proceedings, IEEE International Conference on Communications (ICC), Ottawa, ON, 10-15 June 2012, pp. 1964-1969

21. RS Bhamber, S Fowler, C Braimiotis, A Mellouk, Analytic analysis of LTE/LTE-advanced power saving and delay with bursty traffic. Proceedings, IEEE International Conference on Communications (ICC), Budapest, 9-13 June 2013, pp. 2964-2968

22. S Jin, D Qiao, Numerical analysis of the power saving in 3GPP LTE advanced wireless networks. IEEE Trans. Veh. Technol. 61(4), 1779-1785 (2012)

23. Y-P Yu, K-T Feng, Traffic-based DRX cycles adjustment scheme for 3GPP LTE systems. Proceedings, IEEE Vehicular Technology Conference (VTC Spring), Yokohama, 6-9 May 2012, pp. 1-5

24. SC Jha, AT Koc, R Vannithamby, Optimization of discontinuous reception (DRX) for mobile internet applications over LTE, in Proceedings of IEEE Vehicular Technology Conference (VTC Fall), Quebec City, QC, 3-6 Sept. 2012, pp. 1-5

25. AT Koc, SC Jha, R Vannithamby, M Torlak, Optimizing DRX configuration to improve battery power saving and latency of active mobile applications over LTE-a network. Proceedings, IEEE Wireless Communications and Networking Conference (WCNC), 2013, pp. 568-573

26. SC Jha, AT Koc, R Vannithamby, M Torlak, Adaptive DRX configuration to optimize device power saving and latency of mobile applications over LTE advanced network, in Proceedings of IEEE International Conference on Communications (ICC), 2013, pp. 6210-6214

27. J Wigard, T Kolding, L Dalsgaard, C Coletti, On the user performance of LTE UE power savings schemes with discontinuous reception in LTE. Proceedings, IEEE International Conference on Communications Workshops, 2009, pp. 1-5

28. M Polignano, D Vinella, D Laselva, J Wigard, TB Sorensens, Power savings and QoS impact for VolP application with DRX/DTX feature in LTE. Proceedings, IEEE Vehicular Technology Conference (VTC Spring), 2011, pp. 1-5

29. M Gupta, SC Jha, AT Koc, R Vannithamby, Energy impact of emerging mobile internet applications on LTE network: issues and solutions. IEEE Commun. Mag. 51, 90-97 (2013)

30. S Gao, H Tian, J Zhu, L Chen, A more power-efficient adaptive discontinuous reception mechanism in LTE. Proceedings, IEEE Vehicular Technology Conference (VTC Fall), 2011, pp. 1-5
31. S Fowler, Study on power saving based on radio frame in LTE wireless communication system using DRX. Proceedings, IEEE GLOBECOM Workshops, 2011, pp. 1062-1066

32. PK Gupta, RV Rajakumar, CS Kumar, Energy impact of signalling protocols in 3GPP-LTE and guidelines for savings. Proceedings, Annual IEEE India Conference (INDICON), 2012, pp. 126-130

33. P Frenger, P Moberg, J Malmodin, Y Jading, I Godor, Reducing energy consumption in LTE with cell DTX. Proceedings, IEEE Vehicular Technology Conference (VTC Spring), 2011, pp. 1-5

34. T Adhikary, AK Das, MA Razzaque, MO Rahman, CS Hong, A distributed wake-up scheduling algorithm for base stations in green cellular networks. Proceedings, International Conference on Ubiquitous Information Management and Communication (ICUIMC), Article No.120, 2012

35. F Capozzi, G Piro, LA Grieco, G Boggia, P Camarda, Downlink packet scheduling in LTE cellular networks: key design issues and a survey. IEEE Comm. Surv. Tutor. 15(2), 678-700 (2013)

\section{Submit your manuscript to a SpringerOpen ${ }^{\odot}$ journal and benefit from:}

- Convenient online submission

- Rigorous peer review

- Immediate publication on acceptance

- Open access: articles freely available online

- High visibility within the field

- Retaining the copyright to your article

Submit your next manuscript at $>$ springeropen.com 